\title{
Understanding and Impacting the Practice of Business Process Management
}

\author{
Michael Rosemann \\ Queensland University of Technology, Australia \\ m.rosemann@qut.edu.au
}

This presentation will explore how BPM research can seamlessly combine the academic requirement of rigor with the aim to impact the practice of Business Process Management. After a brief introduction into the research agendas as they are perceived by different BPM communities, two research projects will be discussed that illustrate how empirically-informed quantitative and qualitative research, combined with design science, can lead to outcomes that BPM practitioners are willing to adopt. The first project studies the practice of process modeling using Information Systems theory, and demonstrates how a better understanding of this practice can inform the design of modeling notations and methods. The second project studies the adoption of process management within organizations, and leads to models of how organizations can incrementally transition to greater levels of BPM maturity. The presentation will conclude with recommendations for how the BPM research and practitioner communities can increasingly benefit from each other. 